\title{
Suppressing Dissipation in a Floquet-Hubbard System
}

\author{
Konrad Viebahn๑," Joaquín Minguzzi, Kilian Sandholzer, Anne-Sophie Walter, Manish Sajnani, \\ Frederik Görg, and Tilman Esslinger@ \\ Institute for Quantum Electronics, ETH Zurich, 8093 Zurich, Switzerland
}

(Received 13 March 2020; revised 30 October 2020; accepted 20 November 2020; published 19 March 2021)

\begin{abstract}
The concept of "Floquet engineering" relies on an external periodic drive to realize novel, effectively static Hamiltonians. This technique is being explored in experimental platforms across physics, including ultracold atoms, laser-driven electron systems, nuclear magnetic resonance, and trapped ions. The key challenge in Floquet engineering is to avoid the uncontrolled absorption of photons from the drive, especially in interacting systems in which the excitation spectrum becomes effectively dense. The resulting dissipative coupling to higher-lying modes, such as the excited bands of an optical lattice, has been explored in recent experimental and theoretical works, but the demonstration of a broadly applicable method to mitigate this effect is lacking. Here, we show how two-path quantum interference applied to strongly correlated fermions in a driven optical lattice suppresses dissipative coupling to higher bands and increases the lifetime of double occupancies and spin correlations by 2 to 3 orders of magnitude. Interference is achieved by introducing a weak second modulation at twice the fundamental driving frequency with a definite relative phase. This technique is shown to suppress dissipation in both weakly and strongly interacting regimes of an offresonantly driven Hubbard system, opening an avenue to realizing low-temperature phases of matter in interacting Floquet systems.
\end{abstract}

DOI: 10.1103/PhysRevX.11.011057

\author{
Subject Areas: Atomic and Molecular Physics \\ Condensed Matter Physics \\ Strongly Correlated Materials
}

\section{INTRODUCTION}

Dissipation emerges when a system is coupled to a large number of degrees of freedom in its environment. In periodically driven systems, dissipation thus naturally arises when the low-energy modes are coupled to lossy excited modes by the drive. This form of dissipation presents a formidable challenge to Floquet engineering, in which periodic driving is used to create a host of novel, effectively static Hamiltonians, with ultracold atoms [1-4] and beyond [5-9]. The success of Floquet engineering relies on the existence of a favorable timescale on which a given Floquet Hamiltonian remains valid before heating kicks in and harms the quantum state. Adding interactions to the picture, the effects of unwanted energy absorption are further complicated by a dense excitation spectrum [10-17]. Consequently, the choice of Floquet driving frequency is always a compromise [18]. On the one hand, the Floquet driving frequency should be chosen as high as possible so as to maximize the

*viebahnk@phys.ethz.ch

Published by the American Physical Society under the terms of the Creative Commons Attribution 4.0 International license. Further distribution of this work must maintain attribution to the author(s) and the published article's title, journal citation, and DOI. detuning between the drive and the natural (low-) energy scale of interest $[7,14,18]$. On the other hand, the presence of higher-lying modes of the underlying Hamiltonian, such as energetically higher Bloch bands in an optical lattice [3,4,1826] or in solid state [9], pose a limit on how high the drive frequency can be. In practice, the dissipative coupling to higher bands, due to tunneling within the excited band, inhomogeneities, or subsequent excitations to even higher bands, is the limiting factor in realizing many-body states with Floquet driving. While tailored lattice potentials can be employed to push excited bands to higher frequencies and keep the lowest band dispersive [22], a broadly applicable solution to counteract this form of dissipation has so far not been demonstrated in many-body systems in which the excited modes are densely spaced.

In this paper, we study strongly correlated fermions in an amplitude-modulated optical lattice, i.e., the driven Fermi-Hubbard model, in which the drive couples dissipatively to higher Bloch bands. We demonstrate control of dissipation by introducing a second excitation pathway at twice the fundamental driving frequency and tuning the relative phase [27-30] between the two drives to maximize quantum interference. The performance of this method of dissipation control is quantified by comparing the driven lattice to an equivalent static configuration. 

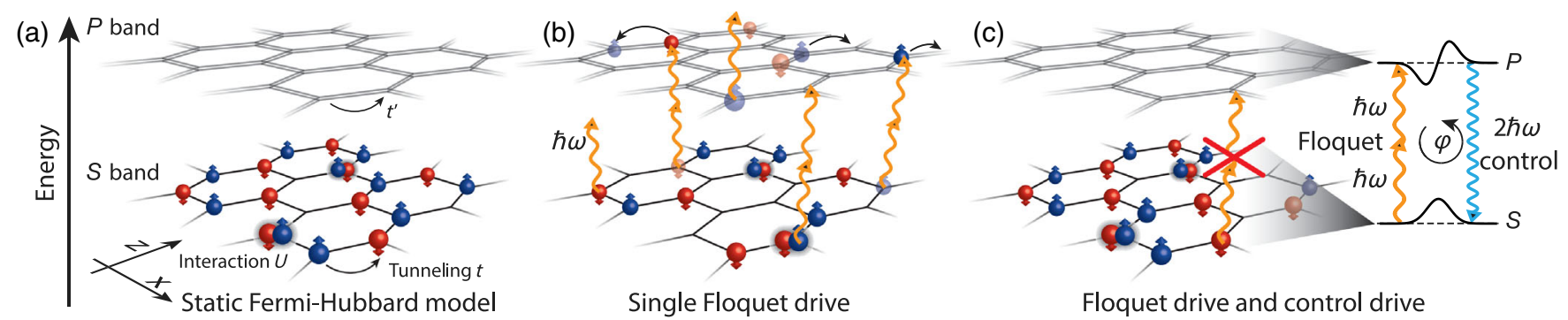

FIG. 1. Two-frequency driving suppresses dissipative coupling to higher bands. (a) Ultracold fermionic atoms in two internal states $\uparrow, \downarrow$ occupy a three-dimensional hexagonal optical lattice ( $y$ direction omitted for clarity). They are described by the ground-band Fermi-Hubbard model with characteristic energies $t$ (tunneling) and $U$ (on-site interaction), while energetically higher-lying bands (tunneling $t^{\prime}$ ) are not populated in the absence of periodic driving. Here, only the fifth band is shown, which originates from hybridized $(P)$ orbitals in the $x$ direction. (b) Periodic driving close to resonance with an interband transition leads to dissipation in the ground-band Fermi-Hubbard system, originating from excited-band tunneling $\left(t^{\prime}\right)$, inhomogeneities, or subsequent excitation to even higher bands. The first relevant resonance is a two-photon process at frequency $\omega$ (shown in orange), whereas the single-photon transition is located at $2 \omega$ (blue). (c) When applying two driving frequencies simultaneously with matched transition strengths, tuning the relative phase $\varphi$ coherently enhances or suppresses dissipative coupling to higher bands.

\section{EXPERIMENTAL SETUP}

The starting point for our experiments is an equal spin mixture of 45000 to 60000 ultracold potassium-40 atoms loaded in an optical lattice, realizing the Fermi-Hubbard model [Fig. 1(a)]. The three-dimensional hexagonal lattice structure [the $y$ direction is not shown in Fig. 1] is characterized by tunnel couplings $\left(t_{x}, t_{y}, t_{z}\right) / h=(340$, $90,106) \mathrm{Hz}$ (see the Appendix C for detailed experimental and theoretical methods) and the Hubbard $U / h$ can be tuned to values between -7 and $7 \mathrm{kHz}$ ( $h$ is Planck's constant). Of the lowest two ( $S$-type) bands of the optical lattice, the ground band is typically populated by $65 \%$ of the atoms. The excited-orbital bands $(P, D, \ldots)$ give rise to a multiband Hubbard model and are not populated in the static case [Fig. 1(a)].

Periodic driving is introduced via amplitude modulation of the lattice depth in the $x$ direction with the waveform

$V_{\bar{X}}(\tau)=V_{0} \times\left[1+A_{\omega} \cos (\omega \tau)+A_{2 \omega} \cos (2 \omega \tau+\varphi)\right]$,

leading predominantly to time-periodic modulation of the tunneling energy $t_{x}$ (Appendix C). In the Floquet framework, the periodic modulation of $t_{x}(\tau)$ could be recast into an effective (static) tunneling $t_{\text {eff }}$ when modulating at resonance with an energy scale of the underlying Hamiltonian $[2,4]$. For instance, modulating $t$ resonantly with both a static site offset and the Hubbard $U$ allows for engineering of anyon-Hubbard models [31]. In general, however, lattice modulation also causes unwanted higherband excitations, particularly for the large driving amplitudes typically required for Floquet engineering $[2,4,31]$. In particular, the appearance of multiphoton resonances [Fig. 1(b)] represents a limitation on the choice of Floquet driving frequency and amplitude [18,20,24].

We characterize single- and multiphoton resonances experimentally by tuning the Hubbard $U$ to zero and recording the excitation spectrum for two different values of the single-frequency modulation amplitude $A_{\omega}$ [Fig. 2(a), $\left.A_{2 \omega}=0\right]$. Here, we modulate the lattice at frequency $\omega$ for a duration of $20 \mathrm{~ms}$ and record the number of atoms in the ground band $\left(N_{\text {ground }}\right)$ by counting atoms in the Brillouin zones after band mapping and time of flight (see Appendix C). The measurement is in good agreement with a numerical solution to the time-dependent Schrödinger equation, taking into account the nonseparable lattice potential in the $x-z$ plane but neglecting any inhomogeneities (Appendix C). Since the modulation is performed on the $t_{x}$ bonds, atoms are predominantly excited to the fifth $(P)$ band which arises from hybridized orbitals in the $x$ direction. Correspondingly, the spectrum in Fig. 2(a) shows pronounced dips in the ground-band population at $24.5 \mathrm{kHz}$ (single-photon resonance) as well as multiphoton resonances at $12.25 \mathrm{kHz}$ (two photon) and $8.17 \mathrm{kHz}$ (three photon). When modulating for a varying amount of time, we never observe a revival of the ground-band population; moreover, the excitations are accompanied by severe atom loss during the modulation (see below). These observations confirm the dissipative nature of the resonance features.

\section{DISSIPATION CONTROL VIA DESTRUCTIVE INTERFERENCE}

In the following, we outline the strategy to mitigate the previously described dissipation process via destructive interference. Modulation at frequency $\omega$ (let us call it the "Floquet" drive) effectively leads to a Rabi coupling to the higher band [19], which in general can be written as a complex number. Now, the key idea is to add a second modulation at $2 \omega$ (the "control" drive) that produces a Rabi coupling of the same magnitude as the Floquet drive but with opposite sign, such that both couplings add up to zero, i.e., interfere destructively. Experimentally, we introduce 

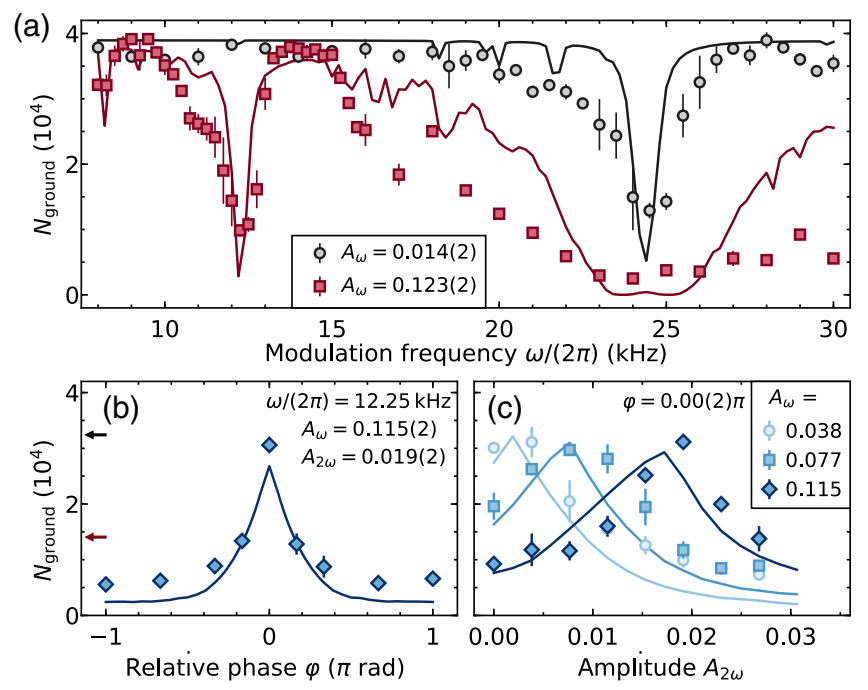

FIG. 2. Optimizing dissipation control in the noninteracting regime. (a) Single-frequency excitation spectrum for noninteracting atoms. The data are obtained by modulating the amplitude of the lattice beam in the $x$ direction for $20 \mathrm{~ms}$ for two relative strengths $A_{\omega}$ of 0.014(2) (gray circles) and 0.123(2) (red squares). Single- and multiphoton resonance features appear at the $S-P$ interband transition at $24.5 \mathrm{kHz}$ and fractions thereof, in agreement with the theoretical calculation (lines) that takes into account the full lattice potential in the $x-z$ plane (see Appendix C). (b) Ground-band population $N_{\text {ground }}$ after $20 \mathrm{~ms}$ of two-frequency modulation [Eq. (1)] as function of the relative phase $\varphi$. A matched excitation strength gives rise to a strong dependence on the relative phase in agreement with $a b$ initio theory (lines). The black (red) arrow shows the ground-band population in the absence of any drive (in the presence of a single drive at $\omega$ ). (c) Ground-band population at $\varphi=0$ as a function of the control amplitude $A_{2 \omega}$ for various Floquet amplitudes $A_{\omega}$. As the Floquet amplitude increases, the optimum control amplitude is shifted to larger values. Error bars to measured data points are the standard error of at least three measurements (see Appendix C).

the control drive with amplitude $A_{2 \omega}$ and vary the relative phase $\varphi$ between the two drives [Fig. 2(b), $A_{\omega}=0.115(2)$, $\left.A_{2 \omega}=0.019(2)\right]$. In the presence of the control drive, the ground-band atom number $N_{\text {ground }}$ shows a strong dependence on $\varphi$, which is direct evidence of phase-only control of the dissipation channel. In particular, $N_{\text {ground }}$ peaks at $\varphi=0.00(1) \pi$, corresponding to a time-reversalsymmetric waveform [Eq. (1)] for which both couplings are real valued but differ by a relative minus sign. This measurement is in good agreement with the theoretical prediction (lines in Fig. 2), as maximal destructive interference leads to a ground-band atom number as high as $30.6(8) \times 10^{3}$, which corresponds to $94 \%$ of the static value [black arrow in Fig. 2(b)]. In contrast, a relative phase of $\pi$ causes depopulation of the ground band with less than $20 \%$ of atoms remaining, compared to $43 \%$ in the absence of the control drive (red arrow). Fixing the relative phase to $\varphi=0$ allows us to achieve destructive interference for increasing values of Floquet amplitude $A_{\omega}$ with the optimum control amplitude shifting to larger values, in good agreement with theory, as is shown in Fig. 2(c). This observation highlights the flexibility of the dissipation control scheme, which is not bound to any particular Floquet amplitude. As the single-photon excitation strength is much larger than the two-photon coupling [24], only a weak control amplitude is necessary to achieve control. In addition, we verify the technique for driving frequencies that are detuned from the higher band resonance (see Appendix B for additional measurements).

\section{DISSIPATION CONTROL IN THE STRONGLY CORRELATED REGIME}

Having demonstrated the control technique in the singleparticle case, we now investigate the influence of interactions on canceling the dissipative coupling to higher bands. The many-body state in the driven Fermi-Hubbard model is experimentally characterized by four different observables, namely, the total atom number $N_{\text {total }}$, groundband population $N_{\text {ground }}$, double occupancy, and nearestneighbour spin correlations (see Appendix C). In order to maximize our sensitivity, we choose to drive our system resonantly with a two-photon higher-band transition with $\omega /(2 \pi)=12.25 \mathrm{kHz}$ with an amplitude of $A_{\omega}=0.115(2)$, giving rise to a strong dissipative coupling. The driving frequency is chosen to be off resonant to any static energy scale such that the lowest-band effective Floquet Hamiltonian is approximately equal to the Fermi-Hubbard model, i.e., $t_{\mathrm{eff}} \simeq t_{x}$. In particular, the Floquet frequency is larger than all probed values of Hubbard $U$, thus precluding density-assisted tunneling or resonant creation of double occupancies, and allowing the quantitative comparison of the static and the driven system [22]. In the experiment, we modulate the system for a varying duration $\tau$ and different values of Hubbard $|U|<\hbar \omega$. These data are then fitted by an exponential decay in order to extract a lifetime. For each measurement, we compare single-frequency modulation $\left[A_{\omega}=0.115(2), A_{2 \omega}=0\right]$ to the two-frequency driving scheme $\left[A_{\omega}=0.115(2), A_{2 \omega}=0.019(2), \varphi=0.00(1) \pi\right]$, as well as the static system. An additional measurement reveals that the optimum relative driving phase $\varphi$ does not depend on the value of Hubbard $U$ (Appendix B).

The measured lifetime of both the total atom number $\tau_{\text {total }}$ and the ground-band population $\tau_{\text {ground }}$ (Fig. 3) shows a strongly increased lifetime in the presence of the control drive compared to the singly driven case. For weak and intermediate interactions $(|U| / h \lesssim 1.5 \mathrm{kHz}=$ bandwidth), the lifetime of the ground-band atom number [Fig. 3(d)] is increased by more than 2 orders of magnitude from $20_{-3}^{+3} \mathrm{~ms}$ to $1.3_{-0.1}^{+0.1} \mathrm{~s}$ at $U / h=1 \mathrm{kHz}$, comparable to the static lifetime. Strong interactions ( $U \gtrsim$ bandwidth) lead to a slight reduction in lifetime in the two-frequency driving protocol compared to the weakly interacting case. Applying dissipation control nevertheless increases the lifetime by 

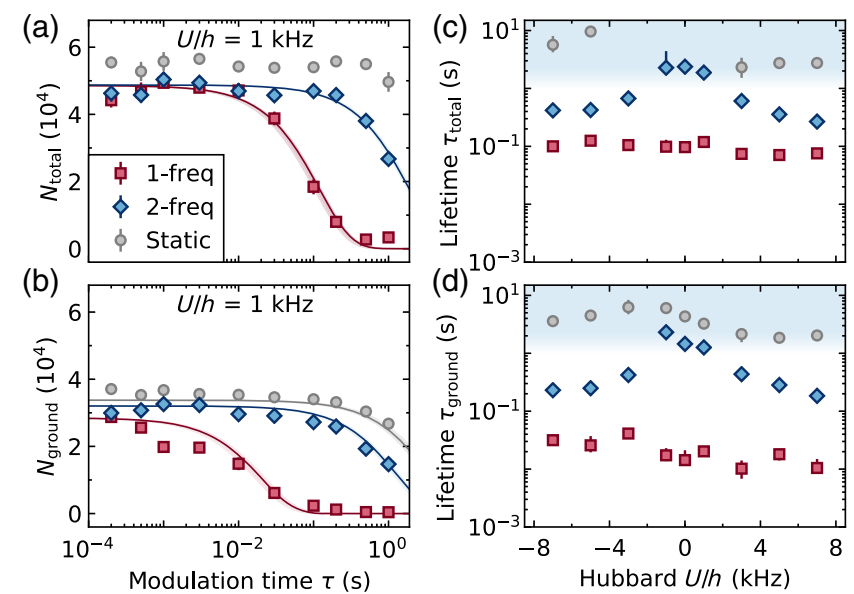

FIG. 3. Effect of interactions on suppressing dissipation. Measured total atom number $N_{\text {total }}$ (a) and ground-band atom number $N_{\text {ground }}$ (b) as a function of the modulation time, to which an exponential decay is fitted (lines). While a single Floquet drive leads to strong atom loss and depopulation of the ground band via higher-band excitations (red points), the presence of the control drive at $2 \omega$ (blue) cancels this dissipation mechanism. (c),(d) Resulting $1 / e$-lifetimes of the total atom number $\left[\tau_{\text {total }},(\mathrm{c})\right]$ and ground-band population $\left[\tau_{\text {ground }},(\mathrm{d})\right]$ as a function of Hubbard $U$. Since the longest measurement time is $1 \mathrm{~s}$, fitted lifetimes longer than this value can be unreliable (blue shaded area). Data points in (a),(b) show the mean and standard error of three individual measurements, whereas the points in (c),(d) result from a leastsquares fit. The shaded areas in (a),(b) and the error bars in (c),(d) show the estimated uncertainty of the fitted value via bootstrapping (Appendix C).

about an order of magnitude. For example, the lifetime of the ground-band atom number at strong repulsive interactions $(U / h=7 \mathrm{kHz})$ shows an 18 -fold increase from $10_{-2}^{+4}$ to $184_{-9}^{+27} \mathrm{~ms}$, corresponding to more than 2000 Floquet driving cycles.

The improved lifetimes in the band-mapping measurements (Fig. 3) strongly suggest that dissipative coupling to higher bands can be efficiently counteracted by adding the control drive, even for large Hubbard $U$. However, the single Floquet drive already leads to heating on timescales shorter than the onset of atoms loss in the ground band (10 to $100 \mathrm{~ms}$ ). In order to investigate this effect, we complement our previous data with measurements of ground-band double occupancy, that is, the fraction of atoms in doubly occupied sites in the lowest Bloch band [22]. This observable is ideally suited to characterize manybody states in the driven Fermi-Hubbard model, and it has been used in the past for an ab initio comparison of our experiment with nonequilibrium dynamical mean-field theory [32]. In the experiment, we start with a certain level of double occupancy in the static lattice set by the value of $U$, assuming a thermally equilibrated state [22,32]. As before, we introduce the modulation for varying amounts of time $\tau$ and then freeze the lattice in order to suppress any further dynamics (see Appendix C). Atoms on (a)
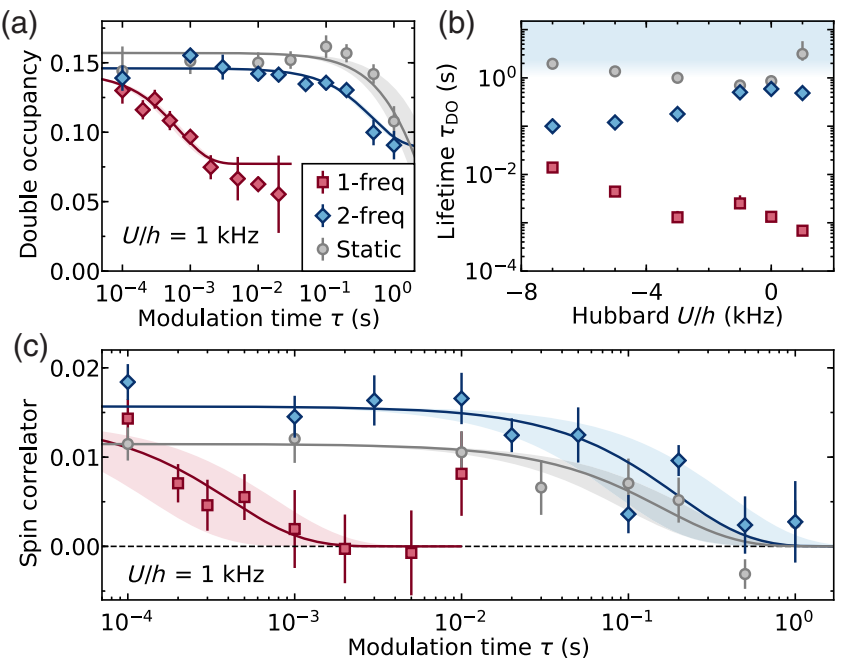

FIG. 4. Suppressing dissipation in the strongly correlated Fermi-Hubbard model. (a) Measured double occupancy as a function of the modulation time. Double occupancy is the fraction of atoms in doubly occupied lattice sites in the ground band. When applying two-frequency control (blue), the level of double occupancy remains almost unchanged up to $1 \mathrm{~s}$, similar to the static case (gray). (b) Fitted exponential lifetimes of double occupancy $\tau_{\mathrm{DO}}$ as a function of Hubbard $U$. For weak and intermediate interactions, the control method (blue) restores the lifetime of double occupancy almost to the static value (gray). We restrict this measurement to values of Hubbard $U / h \leq 1 \mathrm{kHz}$ as the initial value of double occupancy for strong repulsive interactions becomes too low to reliably extract a lifetime. (c) Tracing the nearest-neighbor spin correlator with modulation time for $U / h=1 \mathrm{kHz}$ in the presence of the control drive (blue), compared to the single-frequency case (red). The static reference values are plotted in gray. Data points in (a) and (c) show the mean and standard error of at least three and ten individual measurements, respectively, whereas the plotted lifetimes in (b) result from a least-squares fit. The shaded areas in (a),(c) and the error bars in (b) show the estimated uncertainty of the fitted value via bootstrapping (Appendix C). Since the longest measurement time is $1 \mathrm{~s}$, fitted lifetimes longer than this value can be unreliable [blue shaded area in (b)].

doubly occupied sites are then associated with Feshbach molecules which experience a shifted zero-point on-site energy. As this on-site energy depends on the Bloch band, we are able to spectroscopically resolve double occupancies in the ground band; atoms in higher bands are expelled from the trap during the band-mapping ramp, while atoms in the lowest band remain trapped (Appendix C). The results of this measurement and the extracted lifetimes $\left(\tau_{\mathrm{DO}}\right)$ are shown in Figs. 4(a) and 4(b). We observe a fast drop in double occupancy $\left(\tau_{\mathrm{DO}}=0.7_{-0.1}^{+0.1} \mathrm{~ms}\right.$ for $U / h=$ $1 \mathrm{kHz}$ ) in the single-frequency case, which precedes the ground-band atom loss $\left[\tau_{\mathrm{DO}} \ll \tau_{\text {ground }}=20_{-3}^{+3} \mathrm{~ms}\right.$, Fig. 3(d)]. This observation is consistent with a fast increase in entropy, i.e., heating, which causes the cloud to expand in the trap, resulting in a lower density at the center of the 
trap and reduced double occupancy. In stark contrast, the lifetime of double occupancy is increased by up to a factor of 100 when applying two-frequency control. For example, this lifetime exceeds $1 \mathrm{~s}$ for $U / h=1 \mathrm{kHz}$, which is similar to our static lifetimes, reaching the limit within which we can reliably extract a lifetime (blue shaded areas). This measurement corroborates the previous results for strongly attractive and weakly repulsive interactions [Figs. 3(c) and 3(d)].

While charge dynamics (e.g., double occupancy) in the Fermi-Hubbard model are governed by $t$ and $U$, the lowest hierarchy in the system is the spin exchange energy $4 t^{2} / U$. Therefore, spin correlations are highly susceptible to temperature changes, and we thus expect them to vanish quickly in the Floquet-only driven case. The experimental data are plotted in Fig. 4(c), which show that nearestneighbor spin correlations are indeed destroyed within just a few driving cycles, with a $1 / e$-lifetime of $410_{-280}^{+360} \mu \mathrm{s}$. Remarkably, dissipation control extends the lifetime of nearest-neighbor spin correlations by 3 orders of magnitude to $200_{-110}^{+200} \mathrm{~ms}$, which is within measurement error of the static value $\left(160_{-70}^{+80} \mathrm{~ms}\right)$.

\section{CONCLUSION}

In conclusion, we demonstrate how adding a weak harmonic to the fundamental driving frequency can suppress dissipative coupling to higher-lying modes in a driven manybody system, even in the presence of strong interactions. The comparison of these results to an equivalent static system suggests that low-temperature phases may become accessible even for Floquet Hamiltonians with no static analog, for example, in an anyon-Hubbard model [31]. Numerical calculations on a two-site, two-band Hubbard model (see Appendix A) reveal excellent performance of two-frequency canceling even when driving on resonance with another energy scale, e.g., the Hubbard $U$. In addition to previous experimental evidence [22], these calculations indicate that driving at resonance with an energy scale within the ground band does not greatly influence loss to higher bands. We therefore anticipate the two-frequency method to be applicable to both off-resonant and near-resonant Floquet driving schemes. The theoretical considerations in Appendix A show potential pathways for understanding the two-frequency method in the presence of interactions. Going further, a detailed theoretical analysis, possibly using nonequilibrium dynamical mean-field theory [32], could reveal the precise interference mechanism in the presence of a dense many-body spectrum. One advantage of the twofrequency method is that it results from quantum interference only, and, consequently, the transition to the excited-band state vanishes (in the time-averaged Hamiltonian). Correspondingly, this method does not rely on favorable coherence times of the excited state. However, very large driving amplitudes, which may be necessary to create specific Floquet Hamiltonians, will lead to further resonances and loss channels. These channels could be counteracted by employing additional control frequencies, in the spirit of pulse-shaping techniques in laser-driven molecular dynamics [33]. Moreover, this procedure will depart from the simple Floquet versus control drive picture presented in this work, necessitating an interplay between several driving frequencies along of the lines of an "enriched" drive scheme. Lastly, it should be pointed out that tuneable couplings to higher bands can be an asset, rather than a nuisance, for instance, in designing novel cooling schemes, not only for optical lattices $[19,34]$ but also in condensed matter [35].

\section{ACKNOWLEDGMENTS}

We would like to thank J. Léonard, D. Malz, M. Messer, Y. Murakami, P. Werner, Z. Zhu, and W. Zwerger for discussions and comments on the manuscript. We thank A. Frank for electronic support. This work is partly funded by the SNF (Project No. 182650), NCCR-QSIT, and ERC advanced grant TransQ (Project No. 742579). K. V. is supported by the ETH Zurich Postdoctoral Fellowship program.

\section{APPENDIX A: DISSIPATION CONTROL FOR STRONG INTERACTIONS AND NEAR-RESONANT DRIVING}

In this section, we elaborate on the case of strong Hubbard interaction $U$. Additionally, we cover the case of near-resonant driving in which the drive frequency $\omega$ is close to another energy scale in the underlying Hamiltonian, e.g., the Hubbard $U$. In general, the simple $1 \omega-2 \omega$ canceling scheme works whenever the ground state and the excited orbital can be considered a two-level system. We show how this is indeed the case in the limit of strong interactions and resonant modulation.

\section{Atomic limit}

For the case of complete suppression of tunneling to neighboring sites, we can study the excitation mechanism induced by the driving on a single site corresponding to the "atomic limit." This limit has been studied by Ref. [19], in which an interaction shift $U_{g e}$ for excited orbital was identified, resulting from the $s$-wave scattering between the ground and excited orbital. The value of $U_{g e}$ is roughly half the value of $U_{g g}$, which is the interaction within the lowest orbital (the usual Hubbard $U$ ). Since the coupling of one atom to the higher orbital is the dominant effect (in the case, where $U_{g g}<E$ ), we do not consider the case of two particles occupying the excited orbital (with scattering $U_{e e}$ ). Consequently, there are two types of states that need to be considered for dissipation control in the atomic limit: the singly and doubly occupied site. In the former case, dissipation control applies as in the noninteracting regime [main text and Fig. 5(a)]. In the latter case, the difference in 


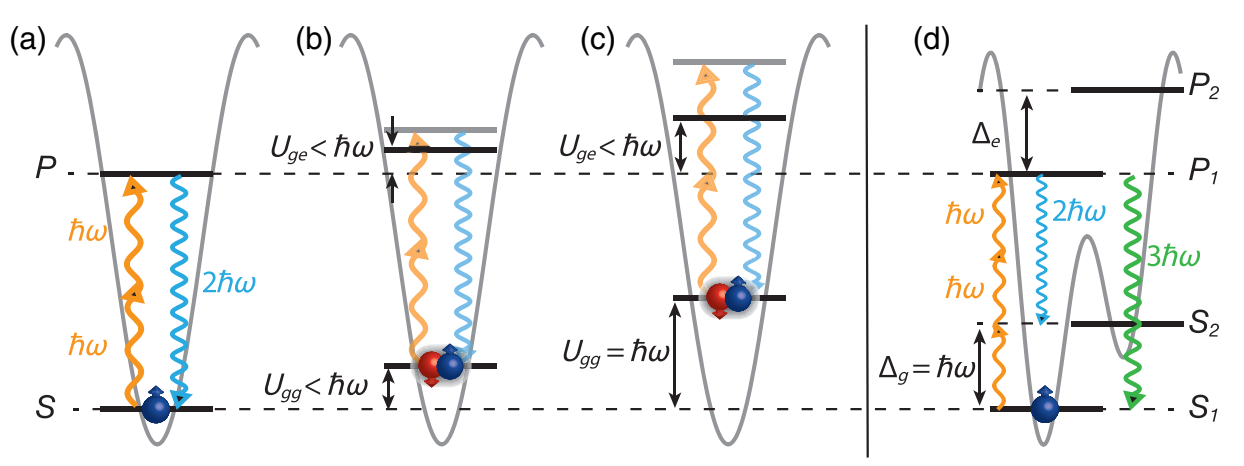

FIG. 5. Dissipation control scheme in the atomic limit for off-resonant and near-resonant driving. This simplified schematic applies to the strongly localized case, the atomic limit, in which the Hubbard $U$ (a)-(c) or, equivalently, a strong site offset $\Delta_{g}$ (d), prohibits tunneling. For strong interactions, two types of states have to be considered: the single occupancy (a) and the double occupancy (b). In the singly occupied case (a), the two-frequency control scheme applies as in the noninteracting regime (as explained in the main text). In the doubly occupied case (b), the distinction between the lower-band $U_{g g}$ and the upper-band $U_{g e}$ interaction leads to a shift in the higher-band resonance [19], reducing the excitation rate to the higher band. The same logic as in (b) applies to near-resonant driving (c) in which $\hbar \omega=U_{g g}$, showing no additional loss features. Near-resonant driving with a static tilt $\Delta_{g}$ in the noninteracting regime (d) can lead to additional resonances, depending on the overlap of the $S_{2}$ and the $P_{1}$ orbitals. If the overlap is small, the control scheme can be used as in (a). If the overlap is large, additional frequencies can be employed to cancel this type of resonance, as shown in (d) for the case of a three-photon resonance from $S_{1}$ to $P_{1}$ and a two-photon resonance from $S_{2}$ to $P_{1}$.

interaction $U_{g g}$ and $U_{g e}$ will shift the excited orbital out of resonance [Fig. 5(b)], as experimentally observed in Ref. [19]. Therefore, both the Floquet drive and control drive will shift out of resonance with the higher band but not affect the dissipation control of single particles. The same applies to the near-resonantly-driven case [Fig. 5(c)], which agrees with Ref. [22], in which no resonant higher-band features were observed in the vicinity of $\hbar \omega \simeq U$. In some driving schemes, however, ground and excited states could experience the same externally imposed energy shift, leading to two possible couplings from the ground to the excited manifold. This situation might occur for driving resonantly to a static tilt $\Delta_{g} \simeq \Delta_{e}$. In Fig. 5(d), we propose a scheme to circumvent additional resonance features by adding a third frequency to the driving protocol.

\section{Beyond the atomic limit}

The atomic limit can be viewed as a good description for deep lattices and strong interactions but cannot account for the more complicated spectrum arising when taking into account correlations between different sites. In this section, we present numerical simulations for a two-site model and demonstrate how the control scheme can be successfully applied to a driving regime resonant to the interaction.

\section{a. Two-site, two-band Fermi-Hubbard model}

We begin by writing down the Hamiltonian that captures the dominating microscopic processes of the Hubbard model on two lattice sites including two separated bands,

$$
\begin{aligned}
\mathcal{H}= & \mathcal{H}_{t}+\mathcal{H}_{U}+\mathcal{H}_{E} \\
= & \sum_{\substack{\sigma \in\{\uparrow, \downarrow\} \\
b \in\{g, e\}}}-t_{b}\left(\hat{c}_{L, \sigma}^{\dagger b} \hat{c}_{R, \sigma}^{b}+\text { H.c. }\right)+E \sum_{\sigma \in\{\uparrow, \downarrow\}}\left(\hat{n}_{L, \sigma}^{e}+\hat{n}_{R, \sigma}^{e}\right) \\
& +\sum_{i \in\{L, R\}}\left[U_{g g} \hat{n}_{i, \downarrow}^{g} \hat{n}_{i, \uparrow}^{g}+U_{e e} \hat{n}_{i, \downarrow}^{e} \hat{n}_{i, \uparrow}^{e}+U_{e g}\left(\hat{n}_{i, \downarrow}^{e} \hat{n}_{i, \uparrow}^{g}+\hat{n}_{i, \downarrow}^{g} \hat{n}_{i, \uparrow}^{e}\right)-U_{e g}\left(\hat{c}_{i, \downarrow}^{\dagger g} \hat{\iota}_{i, \uparrow}^{\dagger e} \hat{c}_{i, \downarrow}^{e} \hat{c}_{i, \uparrow}^{g}+\hat{c}_{i, \uparrow}^{\dagger e} \hat{c}_{i, \downarrow}^{\dagger e} \hat{c}_{i, \uparrow}^{g} \hat{c}_{i, \downarrow}^{g}+\text { H.c. }\right)\right],
\end{aligned}
$$

where $\hat{n}_{i, \sigma}^{b}=\hat{c}_{i, \sigma}^{\dagger b} \hat{c}_{i, \sigma}^{b}$ and $\hat{c}_{i, \sigma}^{\dagger) b}$ is the annihilation (creation) operator for a particle at site $i \in\{L, R\}$ in the band $b \in$ $\{e, g\}$ with spin $\sigma \in\{\uparrow, \downarrow\}$. The tight-binding parameters $t_{e}$ and $t_{g}$ describe the tunneling between neighboring sites in the higher and lower band, respectively. The on-site (contact) interaction between two fermions is represented by $U_{e e}$ (both particles in excited orbital), $U_{e g}$ (one particle in the excited and the other particle in the ground state), and $U_{g g}$ (both particles in the ground state). The interorbital interaction also gives rise to a spin exchange and a pairhopping term $\left(-U_{e g}\right)$. The band gap $E$ describes the energy offset of higher orbital states to the ground band. Here, we consider only a single higher band. For amplitude modulation, processes of order higher than two are strongly suppressed [24], and coupling between opposite parity bands is symmetry forbidden in the localized Wannier basis [23]. Consequently, the most relevant higher band coupled to the ground band is the third band. In this configuration, 
TABLE I. Tight-binding parameters used for the two-site two-band Fermi-Hubbard model [Eq. (A4)]. These include tunneling energies $t_{b}$ in the ground and excited band, the ratios of on-site interactions $U_{b b^{\prime}}$ for two particles in the ground and excited bands, the band gap $E$, the Floquet shaking frequency $\omega$, and the coupling parameters $W_{b, b b^{\prime}}$ of the time-dependent Hamiltonian. The values are obtained using Wannier states of the first (ground) and third (excited) band of a one-dimensional lattice with a depth of $V_{0}=5 E_{R}$.

\begin{tabular}{|c|c|c|c|c|c|}
\hline Quantity & Value (Hz) & Quantity & Value & Quantity & Value \\
\hline$t_{g} / h$ & 291 & $U_{e g} / U_{g g}$ & 0.294 & $W_{g}$ & 0.227 \\
\hline$t_{e} / h$ & 5480 & $U_{e e} / U_{g g}$ & 0.382 & $W_{e}$ & 0.579 \\
\hline$E / h$ & 31340 & & & $W_{g g}$ & 0.017 \\
\hline \multirow[t]{2}{*}{$\omega / 2 \pi$} & 9420 & & & $W_{e g}$ & 0.015 \\
\hline & & & & $W_{e e}$ & 0.011 \\
\hline
\end{tabular}

an additional extended Hubbard term appears coupling lower- and higher-band terms. This term is on the same order as $U_{e g}$ but, since for the case of strong interactions (near-resonant regime) the initial assumption of isolated band states breaks down, we do not consider it here. The tight-binding parameters are estimated from Wannier states calculated for a static onedimensional lattice using the first and third band as the ground and excited band (see Table I).

\section{b. Fock basis and static spectrum}

We consider all 16 possible states using two fermions with opposite spin. The Hamiltonian can be written down in a matrix form using the Fock basis where we use the normal ordering convention

$$
\hat{c}_{R, \uparrow}^{\dagger e} \hat{c}_{R, \uparrow}^{\dagger g} \hat{c}_{L, \downarrow}^{\dagger e} \hat{c}_{L, \uparrow}^{\dagger g}|0\rangle=\left|\begin{array}{l|l}
\downarrow & \uparrow \\
\hdashline & \uparrow
\end{array}\right\rangle .
$$

The Hamiltonian does not couple states with a doubly occupied band and states with only singly occupied bands. The subspace of doubly occupied bands consists of the eight Fock states

$$
\left.\left.\left|\frac{}{\uparrow \mid}\right| \downarrow\right\rangle,\left|\frac{}{\uparrow \downarrow}\right\rangle,\left|\frac{}{\downarrow}\right| \uparrow\right\rangle,\left|\frac{\uparrow}{\mid \uparrow \downarrow}\right\rangle,\left|\frac{\uparrow}{\mid}\right\rangle,\left|\frac{\uparrow \downarrow}{\mid}\right\rangle,\left|\frac{\downarrow}{\mid}\right\rangle,\left|\frac{\uparrow \downarrow}{\mid}\right\rangle,
$$

and the subspace of singly occupied bands involves the Fock states

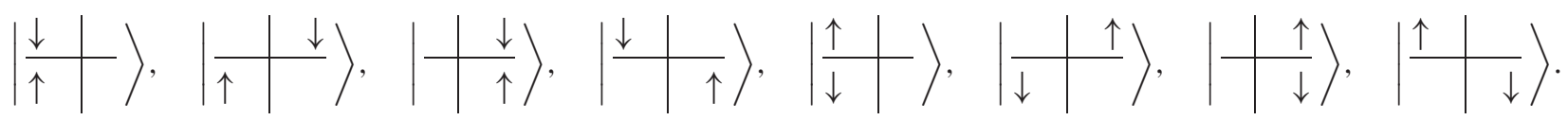

The Hamiltonian in the given basis is written in block diagonal matrix form

$$
H_{0}=\left(\begin{array}{cc}
H_{d} & 0 \\
0 & H_{s}
\end{array}\right)
$$

with the subspace matrices

$$
H_{d}=\left(\begin{array}{cccccccc}
0 & -t_{g} & 0 & -t_{g} & 0 & 0 & 0 & 0 \\
-t_{g} & U_{g g} & t_{g} & 0 & 0 & U_{e g} & 0 & 0 \\
0 & t_{g} & 0 & t_{g} & 0 & 0 & 0 & 0 \\
-t_{g} & 0 & t_{g} & U_{g g} & 0 & 0 & 0 & U_{e g} \\
0 & 0 & 0 & 0 & 2 E & -t_{e} & 0 & -t_{e} \\
0 & U_{e g} & 0 & 0 & -t_{e} & 2 E+U_{e e} & t_{e} & 0 \\
0 & 0 & 0 & 0 & 0 & t_{e} & 2 E & t_{e} \\
0 & 0 & 0 & U_{e g} & -t_{e} & 0 & t_{e} & 2 E+U_{e e}
\end{array}\right),
$$




$$
H_{s}=\left(\begin{array}{cccccccc}
E+U_{e g} & -t_{e} & 0 & t_{g} & -U_{e g} & 0 & 0 & 0 \\
-t_{e} & E & -t_{g} & 0 & 0 & 0 & 0 & 0 \\
0 & -t_{g} & E+U_{e g} & t_{e} & 0 & 0 & -U_{e g} & 0 \\
t_{g} & 0 & t_{e} & E & 0 & 0 & 0 & 0 \\
-U_{e g} & 0 & 0 & 0 & E+U_{e g} & -t_{e} & 0 & t_{g} \\
0 & 0 & 0 & 0 & -t_{e} & E & -t_{g} & 0 \\
0 & 0 & -U_{e g} & 0 & 0 & -t_{g} & E+U_{e g} & t_{e} \\
0 & 0 & 0 & 0 & t_{g} & 0 & t_{e} & E
\end{array}\right) .
$$

We are mainly interested in the lowest-band states whose physics we want to study. In the limit of strong interactions $t_{g} \ll U_{g g}<E$, the eigenstates corresponding to the lowest band states are approximately given by

$$
\begin{aligned}
& |t\rangle=\frac{1}{\sqrt{2}}\left(|\uparrow| \begin{array}{l|l|l} 
& \\
\hline & \downarrow
\end{array} \mid \begin{array}{l|l}
\downarrow & \uparrow
\end{array}\right), \\
& |s\rangle=\frac{1}{\sqrt{2}}\left(\left|\begin{array}{l|l}
\uparrow & \downarrow
\end{array}\right\rangle-\mid \begin{array}{l|l}
\downarrow & \uparrow
\end{array}\right), \\
& \left|D_{ \pm}\right\rangle=\frac{1}{\sqrt{2}}\left(|\uparrow \downarrow|- \pm\left|\frac{}{\uparrow \downarrow}\right\rangle\right) .
\end{aligned}
$$

At zero interaction, the singlet state $|s\rangle$ undergoes an avoided crossing with the $\left|D_{+}\right\rangle$state, which becomes the ground state in the limit of strong attractive interactions (Fig. S2).

\section{c. Including amplitude modulation}

We include amplitude modulation in our system consisting of a Floquet drive (frequency resonant to interaction) and a control drive (frequency resonant to a higher band). The single-particle time-dependent Hamiltonian is given by

$$
\mathcal{H}_{\mathrm{SP}}^{\prime}(\tau)=V_{0}(\alpha \cos (\omega \tau)+\gamma \cos (2 \omega \tau+\phi)) \sin ^{2}\left(k_{l} x\right),
$$

where $\alpha(\gamma)$ is the relative strength of the modulation with frequency $\omega(2 \omega)$. For the second-quantized form, we approximate the Wannier states to be time independent and write the matrix elements as

$$
W_{b b^{\prime}}^{s s^{\prime}}=\left\langle s, b\left|\sin ^{2}\left(k_{l} x\right)\right| s^{\prime}, b^{\prime}\right\rangle,
$$

where $|s, b\rangle$ denotes the Wannier state at site $s \in\{L, R\}$ and band $b \in\{e, g\}$. Because of the symmetry between the left and right site, we can drop the superscript and are left with Wannier overlaps on the same site $W_{b}$ and neighboring sites $W_{b b^{\prime}}$. The resulting Hamiltonian includes timedependent contributions for the tunneling and band-gap energies, as well as an additional interband coupling term

$$
\mathcal{H}(\tau)=\mathcal{H}_{t}(\tau)+\mathcal{H}_{U}+\mathcal{H}_{E}(\tau)+\mathcal{H}^{\prime}(\tau)
$$

with the time-dependent parts

$$
\begin{aligned}
\mathcal{H}_{t}(\tau)= & \sum_{\substack{\sigma \in\{\uparrow \uparrow,\}\} \\
b \in\{g,\}}}\left[-t_{b}+V_{0} W_{b b}(\alpha \cos (\omega \tau)+\gamma \cos (2 \omega \tau+\phi))\right]\left(\hat{c}_{L, \sigma}^{\dagger b} \hat{c}_{R, \sigma}^{b}+\text { H.c. }\right), \\
\mathcal{H}_{E}(\tau)= & {\left[E+V_{0} W_{e}(\alpha \cos (\omega \tau)+\gamma \cos (2 \omega \tau+\phi))\right] \sum_{\sigma, i} \hat{n}_{i, \sigma}^{e} } \\
& +V_{0} W_{g}(\alpha \cos (\omega \tau)+\gamma \cos (2 \omega \tau+\phi)) \sum_{\sigma, i} \hat{n}_{i, \sigma}^{g}, \\
\mathcal{H}^{\prime}(\tau)= & V_{0} W_{e g}(\alpha \cos (\omega \tau)+\gamma \cos (2 \omega \tau+\phi)) \sum_{\sigma, i}\left(\hat{c}_{i, \sigma}^{\dagger e} \hat{c}_{i, \sigma}^{g}+\text { H.c. }\right) .
\end{aligned}
$$

We can write the time-dependent part of Hamiltonian (A4) in the basis defined in Eqs. (A2) and (A3) as the matrix form

$$
V_{0}(\alpha \cos (\omega \tau)+\gamma \cos (2 \omega \tau+\phi))\left(\begin{array}{cc}
W_{1} & W \\
W^{T} & W_{2}
\end{array}\right)
$$

with the coupling matrices 


$$
\begin{aligned}
& W_{1}=\left(\begin{array}{cccccccc}
2 W_{g} & W_{g g} & 0 & W_{g g} & 0 & 0 & 0 & 0 \\
W_{g g} & 2 W_{g} & -W_{g g} & 0 & 0 & 0 & 0 & 0 \\
0 & -W_{g g} & 2 W_{g} & -W_{g g} & 0 & 0 & 0 & 0 \\
W_{g g} & 0 & -W_{g g} & 2 W_{g} & 0 & 0 & 0 & 0 \\
0 & 0 & 0 & 0 & 2 W_{e} & W_{e e} & 0 & W_{e e} \\
0 & 0 & 0 & 0 & W_{e e} & 2 W_{e} & -W_{e e} & 0 \\
0 & 0 & 0 & 0 & 0 & -W_{e e} & 2 W_{e} & -W_{e e} \\
0 & 0 & 0 & 0 & W_{e e} & 0 & -W_{e e} & 2 W_{e}
\end{array}\right) \\
& W_{2}=\left(\begin{array}{cccccccc}
W_{g}+W_{e} & W_{e e} & 0 & -W_{g g} & 0 & 0 & 0 & 0 \\
W_{e e} & W_{g}+W_{e} & W_{g g} & 0 & 0 & 0 & 0 & 0 \\
0 & W_{g g} & W_{g}+W_{e} & -W_{e e} & 0 & 0 & 0 & 0 \\
-W_{g g} & 0 & -W_{e e} & W_{g}+W_{e} & 0 & 0 & 0 & 0 \\
0 & 0 & 0 & 0 & W_{g}+W_{e} & W_{e e} & 0 & -W_{g g} \\
0 & 0 & 0 & 0 & W_{e e} & W_{g}+W_{e} & W_{g g} & 0 \\
0 & 0 & 0 & 0 & 0 & W_{g g} & W_{g}+W_{e} & -W_{e e} \\
0 & 0 & 0 & 0 & -W_{g g} & 0 & -W_{e e} & W_{g}+W_{e}
\end{array}\right), \\
& W=\left(\begin{array}{cccccccc}
0 & W_{e g} & 0 & 0 & 0 & 0 & 0 & W_{e g} \\
W_{e g} & 0 & 0 & 0 & -W_{e g} & 0 & 0 & 0 \\
0 & 0 & 0 & W_{e g} & 0 & W_{e g} & 0 & 0 \\
0 & 0 & W_{e g} & 0 & 0 & 0 & -W_{e g} & 0 \\
0 & W_{e g} & 0 & 0 & 0 & 0 & 0 & W_{e g} \\
W_{e g} & 0 & 0 & 0 & -W_{e g} & 0 & 0 & 0 \\
0 & 0 & 0 & W_{e g} & 0 & W_{e g} & 0 & 0 \\
0 & 0 & W_{e g} & 0 & 0 & 0 & -W_{e g} & 0
\end{array}\right) .
\end{aligned}
$$

The Floquet states of the full time-dependent problem of Eq. (A4) are calculated using exact diagonalization of the Hamiltonian in the extended Sambé space [3]. For the calculations, we truncate the extended space to \pm 11 photon numbers being left with a 368 by 368 matrix for the quasienergy operator.

\section{d. Results}

The energy scales of our model are determined by the depth of the chosen one-dimensional lattice. We use a depth of $V_{0}=5 E_{R}$ and calculate the Wannier states of the static lattice to extract the tight-binding parameters for our model shown in Table I. The Floquet states are then calculated and ordered corresponding to the highest overlap with the static eigenstates. In Fig. 6(a), the full quasienergy spectrum is plotted against the ground-band interaction $U_{g g}$ for a fixed driving frequency $\omega /(2 \pi)=9420 \mathrm{~Hz}$ and strength $\alpha=0.5$. The four ground-band states are highlighted as colored solid lines. At zero interaction, an avoided crossing appears between the $|s\rangle$ and $\left|D_{+}\right\rangle$with gap $4 t_{g}$. When the interaction becomes resonant to the driving frequency, we can observe another avoided crossing between the $|s\rangle$ and $\left|D_{+}\right\rangle$state. A enlargement of this region is provided in Fig. 6(b), corresponding to the small black-boxed region in Fig. 6(a) at $9.5 \mathrm{kHz}$. This avoided crossing resembles the physics of the effective Floquet Hamiltonian in the lowest band [36]. However, the resonant coupling to the higher band creates an additional coupling between the groundband state $\left|D_{-}\right\rangle$to a state $|\vartheta\rangle$ involving both ground- and higher-orbital contributions. This unwanted process can be suppressed using the control drive. In Fig. 6(c), the additional drive with strength $\gamma=0.1$ closes the gap without changing the gap opening between the lowest band states. 

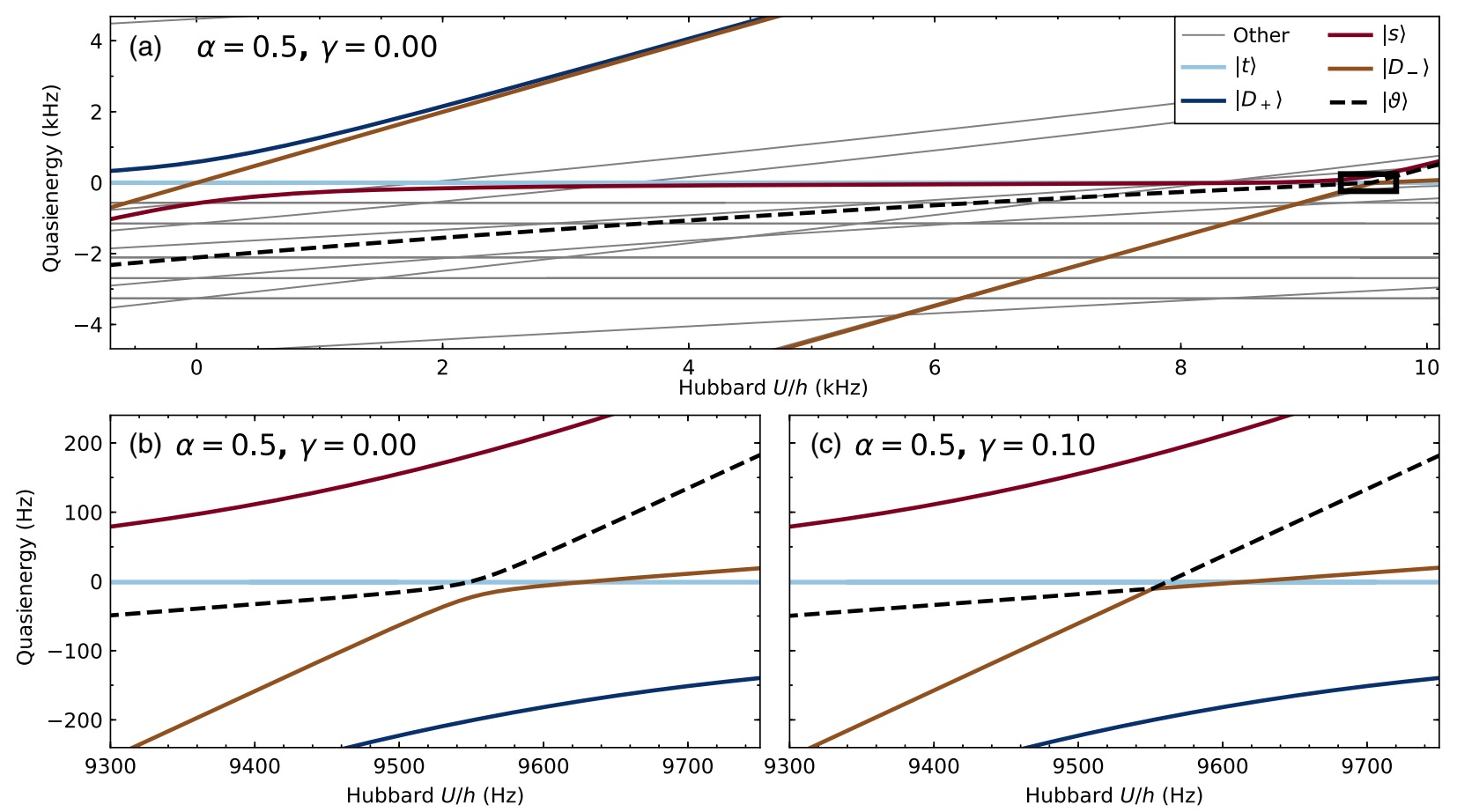

FIG. 6. Quasienergy spectra of the two-site two-band Fermi-Hubbard model (spins $\uparrow, \downarrow$ ) with resonant amplitude modulation. All states that solely occupy ground-band orbitals $\left(\left\langle\hat{n}^{e}\right\rangle=0\right)$ are denoted by thick colored lines. (a) Full quasienergy spectrum (one Floquet zone) for a single-frequency amplitude modulation of strength $\alpha=0.5$ and frequency $\omega /(2 \pi)=9420 \mathrm{~Hz}$. (b) Enlarged spectrum [black box in (a) at $9.5 \mathrm{kHz}$. The Floquet drive couples the $|s\rangle$ state (red) to the $\left|D_{+}\right\rangle$state (blue), leading to an avoided crossing at roughly $U \simeq \hbar \omega$. This resonant effect is accompanied by an additional unwanted avoided crossing of the $\left|D_{-}\right\rangle$ground-band state (light brown) to a state involving both ground- and excited-orbital contributions (black dashed, $|\vartheta\rangle$ ). (c) The addition of a second control drive at $2 \omega$ (strength $\gamma=0.10$ ) decouples the higher-orbital manifold from the Floquet physics in the ground band by closing the gap between ground- and excited-orbital states. The avoided crossing between $|s\rangle$ and $\left|D_{+}\right\rangle$is unaffected by the second drive.

\section{APPENDIX B: ADDITIONAL MEASUREMENTS}

\section{Dissipation control when being detuned from higher-band transitions}

The control drive destructively interferes with the Floquet drive, causing the coupling to the higher band to vanish. Thus, one may ask what happens when the Floquet drive is not exactly on resonance with the higher-band transition. In order to investigate this question, we repeat the measurement from Fig. 2(a) by taking a frequency scan with and without the control drive present. The result is shown in Fig. 7 and shows complete cancellation of the loss feature at all measured frequencies.

\section{Optimum canceling phase for various values of Hubbard $U$}

Interactions modify the excitation spectrum of atoms in the optical lattice. Therefore, one could expect the optimum relative driving phase for dissipation control to vary as a function of the interaction strength. We investigate this effect by measuring the ground-band atom number as a function of the relative driving phase for a broad range of Hubbard $U$ values. The results are displayed in Fig. 8 and

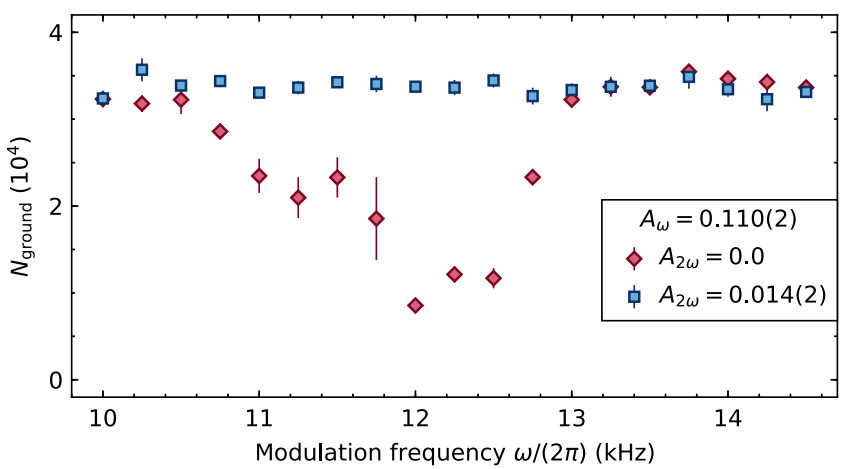

FIG. 7. Dissipation control when being not resonant with higher bands. The lattice is modulated for $20 \mathrm{~ms}$ with an amplitude of $A_{\omega}=0.110(2)$ using the same configuration as in Fig. 2 in the main text. Without the control drive present (red points), the resonant loss to higher bands leads to a strong depopulation of the ground-band atom number $N_{\text {ground }}$. When adding the control drive at $2 \omega$ with an amplitude $A_{2 \omega}=0.014(2)$ and relative driving phase $\varphi=0.00(2)$, keeping the $1: 2$ ratio of frequencies of the Floquet drive and the control drive fixed, destructive interference completely cancels the loss resonance at all measured frequencies (blue points). 


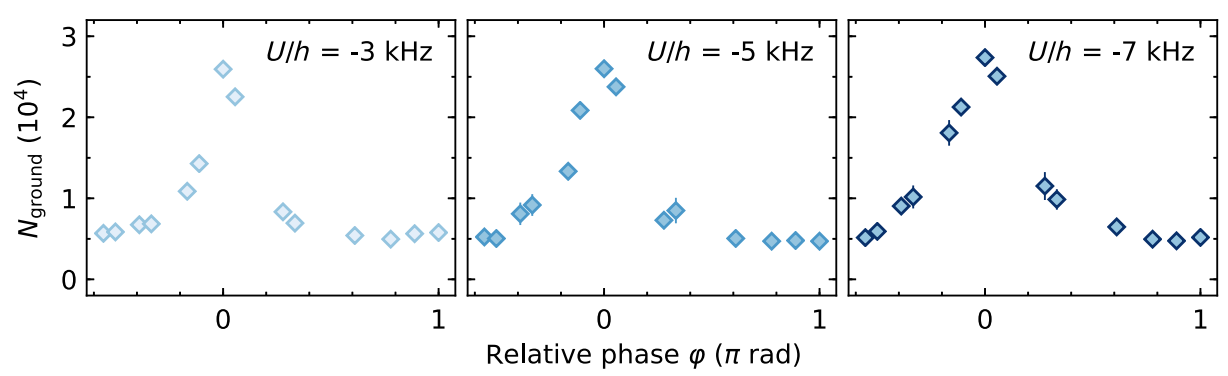

FIG. 8. Effect of interactions on the optimum canceling phase. The measurement of Fig. 2(b) in the main text is repeated for three different values of Hubbard $U$, namely, $-3 \mathrm{kHz}$ (left), $-5 \mathrm{kHz}$ (middle), and $-7 \mathrm{kHz}$ (right). The optimum relative driving phase $\varphi$ does not shift as a function of the interaction strength.

show that the optimum canceling phase does not depend on the value of Hubbard $U$.

\section{APPENDIX C: EXTENDED EXPERIMENTAL AND THEORETICAL METHODS}

\section{General preparation}

To realize the driven Fermi-Hubbard model, we first prepare a gas of ${ }^{40} \mathrm{~K}$ fermionic atoms in the two magnetic sublevels $m_{F}=-9 / 2,-7 / 2$ of the $F=9 / 2$ manifold, which is trapped in a harmonic optical dipole trap. We prepare an incoherent spin-balanced mixture of the two spins by performing many radio-frequency sweeps over the transition resonance between the two states. The cloud is then evaporatively cooled to quantum degeneracy at a scattering length of $a=116(1) a_{0}$, where $a_{0}$ is the Bohr radius. The mean number of atoms in this cloud is $46(2) \times 10^{3}$ at a temperature of $0.09(1) T / T_{F}$. For strong attractive and weak repulsive interactions, we use the $-9 / 2,-7 / 2$ mixture, whereas for strong repulsive interactions, we use a $-9 / 2,-5 / 2$ mixture. The lattice mixture is prepared by applying a Landau-Zener sweep to transfer all atoms from the $-7 / 2$ to the $-5 / 2$ spin state.

We create a three-dimensional optical lattice by retroreflecting four beams of wavelength $\lambda=1064 \mathrm{~nm}$. The resulting lattice potential perceived by the atoms is

$$
\begin{aligned}
V(x, y, z)= & -V_{\bar{X}} \cos ^{2}\left(k_{L} x+\theta / 2\right)-V_{X} \cos ^{2}\left(k_{L} x\right) \\
& -V_{\tilde{Y}} \cos ^{2}\left(k_{L} y\right)-V_{Z} \cos ^{2}\left(k_{L} z\right) \\
& -2 \alpha \sqrt{V_{X} V_{Z}} \cos \left(k_{L} x\right) \cos \left(k_{L} z\right) \cos \varphi_{\mathrm{SL}},
\end{aligned}
$$

where $k_{L}=2 \pi / \lambda$ and $x, y, z$ are the three experimental axes. The lattice depths $V_{\bar{X}, X, \tilde{Y}, Z}$ are measured in units of the recoil energy $E_{R}=h^{2} / 2 m \lambda^{2}$, where $h$ is the Planck constant and $m$ the mass of the atoms. Each lattice depth is independently calibrated by modulating its amplitude in the presence of a ${ }^{40} \mathrm{~K}$ degenerate Fermi cloud. The visibility $\alpha=0.958(8)$ is also calibrated using amplitude modulation on a degenerate ${ }^{40} \mathrm{~K}$ Fermi cloud but in an interfering lattice configuration. The phase $\theta$ that fixes the geometry of the lattice is set to $\theta=1.000(2) \pi$, whereas the superlattice phase $\varphi_{\mathrm{SL}}$ is actively stabilized to $0.00(3) \pi$.

Nonzero temperature leads to a significant population of the second-lowest band which accounts for the difference between $N_{\text {ground }}$ and $N_{\text {total }}$ in Fig. 3 in the main text.

\section{Lattice configurations and modulation}

The potential from Eq. (C1) yields a hexagonal lattice structure, which is characterized by the lowest-band tunneling rates $t_{x}, t_{x, \text { inter }}, t_{y}$, and $t_{z}$. We load the atoms from the optical trap into an intermediate lattice within $200 \mathrm{~ms}$, where the tunneling $t_{x, \text { inter }}$ between dimers remains finite $V_{\bar{X}, X, \tilde{Y}, Z} / E_{R}=[7.89(9), 0.10(2), 9.7(2), 8.2(2)]$. We then linearly ramp up the lattice in $20 \mathrm{~ms}$ to a hexagonal configuration with $V_{\bar{X}, X, \tilde{Y}, Z} / E_{R}=[21.0(3), 3.70(4), 9.7(2)$, 6.81(8)] in which the modulation is performed. Here, the resulting tunneling energies are $\left[t_{x}, t_{x, \text { inter }}, t_{y}, t_{z}\right] / h=$ $[340(50), 0.8(1), 90(4), 106(7)] \mathrm{Hz}$, where the interdimer tunneling $t_{x, \text { inter }}$ is negligibly small. To implement the periodic drive in the system, we modulate the intensity of the noninterfering $\bar{X}$ lattice beam by applying a sinusoidal signal to the rf power of an acousto-optic modulator resulting in a modulation of the lattice depth. We obtain a periodically varying lattice depth of the form $V_{\bar{X}}(\tau)=V_{\bar{X}}\left(1+A_{\omega} \sin (\omega \tau)\right)$, where $\omega$ corresponds to the Floquet driving frequency and $A_{\omega}$ to the relative amplitude of the drive. The lattice modulation leads to a modulation of all Hubbard parameters. However, the influence of the modulation on $t_{z}$ remains smaller than $13 \%$ and that on $U$ smaller than $5 \%$. Such a periodic modulation will also introduce transitions to higher bands, specifically to the fifth band, which results from hybridized orbitals in the $x$ direction. While in separable square lattices the coupling resulting from amplitude modulation between $S$ and $P$ bands reduces to zero at the band center and at the band edges [34], for other quasimomentum states this process is in general allowed. In our nonseparable lattice, in particular, the process is allowed, corroborating the agreement between measurement and ab initio theory.

To compensate for these losses, we add an additional "control" contribution to the drive $A_{2 \omega} \sin (2 \omega t+\varphi)$ at 
twice the frequency. The amplitude and phase of the drive are calibrated by recording the intensity of the lattice beam and performing a fast Fourier transform on the signal. The remaining systematic deviations are $2^{\circ}=0.01 \pi$ in the single-frequency driving phase and 0.002 in the amplitude; statistical deviations are smaller than these values. In all measurements presented in the paper, the amplitude of the modulation is ramped to its final value within two periods of the fundamental modulation frequency $\omega$ and suddenly switched off after the duration of the modulation $\tau$.

\section{Detection methods}

The experimental realization of the Fermi-Hubbard model is characterized by four observables: atom number and band population in Fig. 3, as well as double-occupancy fraction and spin correlations in Fig. 4.

\section{a. Band-mapping detection}

The atom number and band population are obtained through a detection method called band mapping. Directly after the modulation, we ramp down the optical lattice slowly enough for the atoms to stay adiabatically in their band but fast enough to avoid redistribution between bands. We thereby map quasimomentum to real momentum. We do this by an exponential ramp to zero of the lattice depth within $500 \mu \mathrm{s}$. After $500 \mu \mathrm{s}$, we switch off the homogeneous magnetic field and trapping potential and allow for 15-ms time of flight (TOF) to map momentum onto position and thereby resolve the Brillouin zones in real space. We then take an absorption image of the expanded cloud. To assess the distribution of atoms among bands, we divide the image into zones for the different bands and integrate the atomic density in each zone. The center position of the first Brillouin zone (BZ) is determined by fitting a Gaussian to a ${ }^{40} \mathrm{~K}$ cloud released adiabatically from the trap. The size of the BZ is determined by a separate calibration method where we use a ${ }^{87} \mathrm{Rb}$ condensate and flash the lattice: The $2 \hbar k_{L}$ diffraction peaks yield the edges of the first zone and are corrected by a factor $87 / 40$ due to the mass difference between ${ }^{87} \mathrm{Rb}$ and ${ }^{40} \mathrm{~K}$.

\section{b. Stern-Gerlach detection}

The measurement of double-occupancy fractions and spin correlations requires another detection method. We release the atoms from the lattice within $100 \mathrm{~ms}$ : longer than the $500 \mu$ s from the band mapping to allow for the atoms to redistribute into Gaussian cloud shapes and for atoms in higher bands to be lost from the trap but still short enough to avoid atom loss. Also, these observables require a spin-resolved measurement: To distinguish between the different Zeeman sublevels, we apply a short magnetic field gradient which leads to a separation of the spin states during TOF of $8 \mathrm{~ms}$.

\section{c. Double-occupancy detection}

Directly after the modulation, we freeze the dynamics through a quench to a deep cubic "freeze" lattice $V_{\bar{X}, X, \tilde{Y}, Z}=$ $[30.6(5), 0.0,39.9(2), 29.6(8)]$ within $100 \mu \mathrm{s}$. We then ramp the magnetic field over the $-7 / 2,-9 / 2$ Feshbach resonance at $B=202.1 \mathrm{G}$ and spectroscopically resolve the interaction shift by radio-frequency radiation. As a result, only the atoms sitting in pairs on a lattice site are transferred to another spin state. For the $-7 / 2,-9 / 2$ mixture, the atoms in the $-7 / 2$ are transferred to the $-5 / 2$ state, while for the $-5 / 2,-9 / 2$ mixture, atoms in the $-5 / 2$ are transferred to the $-7 / 2$ state. These Zeeman sublevels are then detected with the previously described Stern-Gerlach method.

The detection efficiency of double occupancy is independently calibrated to be $1.00(1)$ in the simple-cubic lattice and 1.04(3) in the checkerboard configuration (see below). The calibration involves measuring the double-occupancy fraction as described above and comparing it to an alternative method that independently measures the total atom number and then only the singly occupied sites (by removing the double occupancies in a cleaning procedure, as described below). The difference between the total atom number and single occupancies is plotted against the measured double occupancy as a function of the total atom number. These data are fitted with a straight line whose slope is the efficiency stated above in the two lattice configurations.

\section{d. Spin-correlations detection}

This measurement scheme is described in detail in Ref. [37]. In this paper, we use only the $-7 / 2,-9 / 2$ mixture in the spin-correlations measurement. As for the double-occupancy detection, we start by ramping the lattice to a cubic freeze lattice. We then eliminate the double occupancies: Through consecutive radio-frequency Landau-Zener sweeps, we transfer the atoms in the $-7 / 2$ to the $-3 / 2$ spin state. In doubly occupied sites, the $-3 / 2$, $-9 / 2$ mixture is very short-lived, and therefore lost from the trap. The remaining single atoms in the $-3 / 2$ state are transferred back to the $-7 / 2$ state with two consecutive radio-frequency Landau-Zener sweeps. The nearestneighbor spin correlator along $t_{x}$ can be written as

$$
\begin{aligned}
\text { spin correlator } & =-\frac{1}{N} \sum_{\mathbf{i}}\left[\left\langle\hat{S}_{\mathbf{i}}^{x} \hat{S}_{\mathbf{i}+\mathbf{e}_{x}}^{x}\right\rangle+\left\langle\hat{S}_{\mathbf{i}}^{y} \hat{S}_{\mathbf{i}+\mathbf{e}_{x}}^{y}\right\rangle\right] \\
& =\frac{\text { singlet fraction }- \text { triplet fraction }}{2},
\end{aligned}
$$

where $\hat{S}_{i}$ is the standard vector spin operator on lattice site $\mathbf{i}$, the sum runs over all two-site unit cells, $\mathbf{e}_{x}$ is the unit vector in the $x$ direction, and $N$ is the total number of atoms in the ground band. Therefore, full antiferromagnetic correlations along $t_{x}$ would result in a spin correlator of 1. In order to measure the singlet and triplet fraction, we 
apply a magnetic field gradient which leads to an oscillation between the two populations. We measure these populations at the two extrema of the oscillations (4.2 and $7.8 \mathrm{~ms}$ ) and infer the spin correlations from the difference. To do so, we ramp the lattice to a checkerboard configuration $V_{\bar{X}, X, \tilde{Y}, Z}=[0.0,29.6(5), 39.9(2), 29.6(8)]$ thereby merging adjacent sites with singlets and triplets. Because of the Pauli exclusion principle, the triplets are then converted to one atom in the lowest and another in the first excited band. The singlets, on the other hand, form double occupancies in the lowest band. These single or double occupancies in the lowest band can be detected with the same method as described previously for double occupancies. To normalize these fractions, we determine the total number of atoms $N$ including double occupancies in a separate measurement.

\section{Lifetime and band population fits}

To extract a characteristic timescale for the band population transfer and lifetimes for the atom number, doubleoccupancy fraction, and spin correlations, we use an exponential decay function

$$
P(\tau)=P_{0} \exp \left(-\tau / \tau_{P}\right),
$$

where $P_{0}$ corresponds to the initial value of the observable, $\tau$ is the modulation time, and $\tau_{P}$ the fitted lifetime of the observable. The data points and corresponding error bars as a function of the modulation duration correspond to the mean value and standard error from three [Figs. 3(a), 3(b), and 4(a)] to ten [Fig. 4(c)] independent measurements. The curves in these figures correspond to the result of fitting Eq. (C3) to all measured values. To obtain an estimate on the uncertainty of the lifetime, we use two different sampling methods. For the double-occupancy fraction, atom number, and ground-band population [Figs. 3, 4(a), and 4(b)], we use a method called bootstrapping: We randomly resample values from the measured points and fit the exponential decay function to this dataset. For the spin correlations, we assume a normal distribution around the mean measured value for each modulation time. We then sample random values from these distributions and apply an exponential fit to the resulting dataset. We repeat both methods 500 times while varying the initialization parameters by plus minus 10\%: $P_{0}$ and $\tau_{P}$ for the atom number and band population and only $\tau_{P}$ for the double-occupancy fraction and spin correlations $\left(P_{0}\right.$ is then fixed to the initial value from the fit on all measured data points). We plot the mean standard deviation of the distribution of fitted parameters as the shaded area in Figs. 3(a), 3(b), 4(a), and 4(c) and as asymmetric error bars in Figs. 3(c), 3(d), and 4(b).

\section{Band-transfer calculations}

We calculate the band structure of our lattice and use the results to determine the expected population transfer between bands induced by the drive. We extend the methods described in Ref. [24] to 2D lattices. In brief, we start with the time-dependent Hamiltonian where we include the modulation as a periodic relative modulation of the lattice depth:

$$
\mathcal{H}=\frac{\mathbf{p}^{2}}{2 \mu}+V(x, y, z, \tau),
$$

where the lattice potential is similar to Eq. (C1). The mass of the atom is $\mu$. The modulation is implemented by multiplying the noninterfering lattice depth in the $x$ direction with the time-dependent waveform of the modulation:

$V_{\bar{X}}(\tau)=V_{\bar{X}} \times\left[1+A_{\omega} \sin (\omega \tau)+A_{2 \omega} \sin (2 \omega \tau+\varphi)\right]$.

The modulation in the $x$ direction predominantly affects the dynamics in the $x-z$ plane, and we therefore neglect the $y$ direction in all subsequent calculations. To simplify further, we apply a coordinate transformation by rotating our frame by $45^{\circ}$ :

$$
x^{\prime}=\frac{x+z}{\sqrt{2}}, \quad z^{\prime}=\frac{z-x}{\sqrt{2}} .
$$

The Bloch waves of the static Hamiltonian can be decomposed into their Fourier components:

$$
\Psi_{q_{x^{\prime}}, q_{z^{\prime}}}^{n}\left(x^{\prime}, z^{\prime}\right)=\sum_{l, m=-\infty}^{+\infty} c_{l, m}^{n}\left(q_{x^{\prime}}, q_{z^{\prime}}\right) e^{i\left(\left(2 l k_{L}+q_{x^{\prime}}\right) x^{\prime}+\left(2 m k_{L}+q_{z^{\prime}}\right) z^{\prime}\right)} .
$$

Expressing the Hamiltonian in the basis of these coefficients $c_{l, m}^{n}\left(q_{x^{\prime}}, q_{z^{\prime}}\right)$, we can rewrite it as

$$
\mathcal{H}_{l, l^{\prime}, m, m^{\prime}}= \begin{cases}\frac{\hbar^{2}}{2 \mu}\left[2\left(\frac{q_{x^{\prime}}}{2}+l k_{L}\right)^{2}+2\left(\frac{q_{z^{\prime}}}{2}+m k_{L}\right)^{2}\right] & \text { for } l=l^{\prime} \text { and } m=m^{\prime}, \\ -\frac{\alpha}{2} \sqrt{V_{X} V_{Z}} \cos (\phi) & \text { for }\left(\left|l-l^{\prime}\right|=1 \text { and } m=m^{\prime}\right) \text { or }\left(l=l^{\prime} \text { and }\left|m-m^{\prime}\right|=1\right), \\ -\frac{V_{Z}}{4} & \text { for } l=l^{\prime} \pm 1 \text { and } m=m^{\prime} \pm 1, \\ -\frac{V_{\bar{X}}(\tau)}{4}-\frac{V_{X}}{4} e^{ \pm i \theta} & \text { for } l=l^{\prime} \pm 1 \text { and } m=m^{\prime} \mp 1, \\ 0 & \text { otherwise. }\end{cases}
$$


We sample the positive quadrant of $\left(q_{x}, q_{z}\right)$ in steps of $0.25 \times k_{L}$. For each $q$ vector, we integrate the timedependent Schrödinger equation for a finite number of evenly spaced steps in time up to $\Delta \tau$, which corresponds to the modulation time. We assume that the atoms are initially in the lowest band and evolve that state in time. For each time step, we calculate the overlap of the time-evolved state with the static eigenstate of the ground band. The curves in Figs. 2(a) and 3 are obtained by taking the minimum overlap for each quasimomentum and averaging over the entire Brillouin zone.

The Hubbard parameters $t$ and $U$ are numerically calculated from the Wannier functions of the lattice potential, which we obtain from band-projected position operators [38].

[1] N. Goldman and J. Dalibard, Periodically Driven Quantum Systems: Effective Hamiltonians and Engineered Gauge Fields, Phys. Rev. X 4, 031027 (2014).

[2] M. Bukov, L. D’Alessio, and A. Polkovnikov, Universal High-Frequency Behavior of Periodically Driven Systems: From Dynamical Stabilization to Floquet Engineering, Adv. Phys. 64, 139 (2015).

[3] M. Holthaus, Floquet Engineering with Quasienergy Bands of Periodically Driven Optical Lattices, J. Phys. B 49, 013001 (2016).

[4] A. Eckardt, Colloquium: Atomic Quantum Gases in Periodically Driven Optical Lattices, Rev. Mod. Phys. 89, 011004 (2017).

[5] T. Oka and S. Kitamura, Floquet Engineering of Quantum Materials, Annu. Rev. Condens. Matter Phys. 10, 387 (2019).

[6] F. Harper, R. Roy, M. S. Rudner, and S. L. Sondhi, Topology and Broken Symmetry in Floquet Systems, Annu. Rev. Condens. Matter Phys. 11, 345 (2020).

[7] P. Peng, C. Yin, X. Huang, C. Ramanathan, and P. Cappellaro, Floquet Prethermalization in Dipolar Spin Chains, Nat. Phys. (2021), https://doi.org/10.1038/s41567020-01120-Z.

[8] P. Kiefer, F. Hakelberg, M. Wittemer, A. Bermúdez, D. Porras, U. Warring, and T. Schaetz, Floquet-Engineered Vibrational Dynamics in a Two-Dimensional Array of Trapped Ions, Phys. Rev. Lett. 123, 213605 (2019).

[9] Y. H. Wang, H. Steinberg, P. Jarillo-Herrero, and N. Gedik, Observation of Floquet-Bloch States on the Surface of a Topological Insulator, Science 342, 453 (2013).

[10] M. Reitter, J. Näger, K. Wintersperger, C. Sträter, I. Bloch, A. Eckardt, and U. Schneider, Interaction Dependent Heating and Atom Loss in a Periodically Driven Optical Lattice, Phys. Rev. Lett. 119, 200402 (2017).

[11] K. Singh, C. J. Fujiwara, Z. A. Geiger, E. Q. Simmons, M. Lipatov, A. Cao, P. Dotti, S. V. Rajagopal, R. Senaratne, T. Shimasaki, M. Heyl, A. Eckardt, and D. M. Weld, Quantifying and Controlling Prethermal Nonergodicity in Interacting Floquet Matter, Phys. Rev. X 9, 041021 (2019).

[12] T. Boulier, J. Maslek, M. Bukov, C. Bracamontes, E. Magnan, S. Lellouch, E. Demler, N. Goldman, and
J. V. Porto, Parametric Heating in a $2 D$ Periodically Driven Bosonic System: Beyond the Weakly Interacting Regime, Phys. Rev. X 9, 011047 (2019).

[13] K. Wintersperger, M. Bukov, J. Näger, S. Lellouch, E. Demler, U. Schneider, I. Bloch, N. Goldman, and M. Aidelsburger, Parametric Instabilities of Interacting Bosons in Periodically Driven 1D Optical Lattices, Phys. Rev. X 10, 011030 (2020).

[14] A. Rubio-Abadal, M. Ippoliti, S. Hollerith, D. Wei, J. Rui, S. L. Sondhi, V. Khemani, C. Gross, and I. Bloch, Floquet Prethermalization in a Bose-Hubbard System, Phys. Rev. X 10, 021044 (2020).

[15] T. Kuwahara, T. Mori, and K. Saito, Floquet-Magnus Theory and Generic Transient Dynamics in Periodically Driven Many-Body Quantum Systems, Ann. Phys. (Amsterdam) 367, 96 (2016).

[16] R. Moessner and S. L. Sondhi, Equilibration and Order in Quantum Floquet Matter, Nat. Phys. 13, 424 (2017).

[17] A. Eckardt and M. Holthaus, Avoided-Level-Crossing Spectroscopy with Dressed Matter Waves, Phys. Rev. Lett. 101, 245302 (2008).

[18] G. Sun and A. Eckardt, Optimal Frequency Window for Floquet Engineering in Optical Lattices, Phys. Rev. Research 2, 013241 (2020).

[19] W. S. Bakr, P. M. Preiss, M. E. Tai, R. Ma, J. Simon, and M. Greiner, Orbital Excitation Blockade and Algorithmic Cooling in Quantum Gases, Nature (London) 480, 500 (2011).

[20] M. Weinberg, C. Ölschläger, C. Sträter, S. Prelle, A. Eckardt, K. Sengstock, and J. Simonet, Multiphoton Interband Excitations of Quantum Gases in Driven Optical Lattices, Phys. Rev. A 92, 043621 (2015).

[21] N. Fläschner, M. Tarnowski, B. S. Rem, D. Vogel, K. Sengstock, and C. Weitenberg, High-Precision Multiband Spectroscopy of Ultracold Fermions in a Nonseparable Optical Lattice, Phys. Rev. A 97, 051601(R) (2018).

[22] M. Messer, K. Sandholzer, F. Görg, J. Minguzzi, R. Desbuquois, and T. Esslinger, Floquet Dynamics in Driven Fermi-Hubbard Systems, Phys. Rev. Lett. 121, 233603 (2018).

[23] C. Cabrera-Gutiérrez, E. Michon, M. Arnal, G. Chatelain, V. Brunaud, T. Kawalec, J. Billy, and D. Guéry-Odelin, Resonant Excitations of a Bose Einstein Condensate in an Optical Lattice, Eur. Phys. J. D 73, 170 (2019).

[24] C. Sträter and A. Eckardt, Interband Heating Processes in a Periodically Driven Optical Lattice, Z. Naturforsch. A 71, 909 (2016).

[25] A. Keleş, E. Zhao, and W. V. Liu, Effective Theory of Interacting Fermions in Shaken Square Optical Lattices, Phys. Rev. A 95, 063619 (2017).

[26] A. Quelle and C. M. Smith, Resonances in a Periodically Driven Bosonic System, Phys. Rev. E 96, 052105 (2017).

[27] M. Schiavoni, L. Sanchez-Palencia, F. Renzoni, and G. Grynberg, Phase Control of Directed Diffusion in a Symmetric Optical Lattice, Phys. Rev. Lett. 90, 094101 (2003).

[28] C. Zhuang, C. R. Paul, X. Liu, S. Maneshi, L. S. Cruz, and A. M. Steinberg, Coherent Control of Population Transfer between Vibrational States in an Optical Lattice via Two-Path Quantum Interference, Phys. Rev. Lett. 111, 233002 (2013). 
[29] L. Niu, D. Hu, S. Jin, X. Dong, X. Chen, and X. Zhou, Excitation of Atoms in an Optical Lattice Driven by Polychromatic Amplitude Modulation, Opt. Express 23, 10064 (2015).

[30] F. Görg, K. Sandholzer, J. Minguzzi, R. Desbuquois, M. Messer, and T. Esslinger, Realization of Density-Dependent Peierls Phases to Engineer Quantized Gauge Fields Coupled to Ultracold Matter, Nat. Phys. 15, 1161 (2019).

[31] L. Cardarelli, S. Greschner, and L. Santos, Engineering Interactions and Anyon Statistics by Multicolor LatticeDepth Modulations, Phys. Rev. A 94, 023615 (2016).

[32] K. Sandholzer, Y. Murakami, F. Görg, J. Minguzzi, M. Messer, R. Desbuquois, M. Eckstein, P. Werner, and T. Esslinger, Quantum Simulation Meets Nonequilibrium Dynamical Mean-Field Theory: Exploring the Periodically Driven, Strongly Correlated Fermi-Hubbard Model, Phys. Rev. Lett. 123, 193602 (2019).

[33] D. G. Kuroda, C. P. Singh, Z. Peng, and V. D. Kleiman, Mapping Excited-State Dynamics by Coherent Control of a
Dendrimer's Photoemission Efficiency, Science 326, 263 (2009).

[34] M. Arnal, V. Brunaud, G. Chatelain, C. Cabrera-Gutiérrez, E. Michon, P. Cheiney, J. Billy, and D. Guéry-Odelin, Evidence for Cooling in an Optical Lattice by Amplitude Modulation, Phys. Rev. A 100, 013416 (2019).

[35] P. Werner, M. Eckstein, M. Müller, and G. Refael, LightInduced Evaporative Cooling of Holes in the Hubbard Model, Nat. Commun. 10, 5556 (2019).

[36] R. Desbuquois, M. Messer, F. Görg, K. Sandholzer, G. Jotzu, and T. Esslinger, Controlling the Floquet State Population and Observing Micromotion in a Periodically Driven TwoBody Quantum System, Phys. Rev. A 96, 053602 (2017).

[37] D. Greif, T. Uehlinger, G. Jotzu, L. Tarruell, and T. Esslinger, Short-Range Quantum Magnetism of Ultracold Fermions in an Optical Lattice, Science 340, 1307 (2013).

[38] T. Uehlinger, G. Jotzu, M. Messer, D. Greif, W. Hofstetter, U. Bissbort, and T. Esslinger, Artificial Graphene with Tunable Interactions, Phys. Rev. Lett. 111, 185307 (2013). 